\title{
Langerhans cell histiocytosis shows distinct cytoplasmic expression of major histocompatibility class II antigens
}

\author{
Lucas Redd ${ }^{1}$ - Monika Schmelz ${ }^{1}$ W. Richard Burack ${ }^{2}$. James R. Cook ${ }^{3}$. \\ Antony W. Day ${ }^{4} \cdot$ Lisa Rimsza $^{5}$
}

Received: 13 November 2015 / Accepted: 29 February 2016/Published online: 10 March 2016

(C) Springer-Verlag Berlin Heidelberg 2016

\begin{abstract}
Langerhans cell histiocytosis ( $\mathrm{LCH})$ is a monoclonal proliferation of antigen presenting cells (APC). In benign APCs, antigen loading occurs in the major histocompatibility class II (MHCII)-lysosomal compartment of the endocytic pathway followed by transport to the cell surface upon antigen stimulation. The pattern of MHCII expression in $\mathrm{LCH}$ is not well characterized. The cellular localization of MHCII was determined using immunohistochemistry (IHC). Staining pattern for the representative MHCII molecule, HLA-DR, (cell surface, cytoplasmic granular, or cytoplasmic globular), and intensity ( 0 to $3+$ ) was recorded for normal tissues and 44 LCH samples along with available clinicopathologic features. Results were confirmed with a different antibody to confirm the appearance. In the normal tissue survey, strong HLA-DR cell surface expression was present on APCs, benign B cells, and some $T$ cells. A granular cytoplasmic staining pattern (without surface expression) was seen in benign Langerhans cells (LCs) in the skin and histiocytes. Strikingly, all $44 \mathrm{LCH}$ samples demonstrated both
\end{abstract}

Lisa Rimsza

rimsza.lisa@mayo.edu

1 Department of Pathology, University of Arizona, Tucson, AZ, USA

2 Department of Pathology, University of Rochester, Rochester, NY, USA

3 Pathology and Laboratory Medicine Institute, Cleveland Clinic, Cleveland, OH, USA

4 Department of Molecular and Cellular Biology, University of Arizona, Tucson, AZ, USA

5 Department of Laboratory Medicine and Pathology, Mayo Clinic, 13400 East Shea Boulevard, CRB 02-112, Scottsdale, AZ 85259, USA cytoplasmic granular and an unusual "globular" staining pattern with no surface staining. This is the first report of a highly specific HLA-DR staining pattern in LCH detected by IHC. The cytoplasmic perinuclear globular localization of MHCII may possibly be useful in diagnostics and may result from an immature/antigen-naïve differentiation state of the neoplastic cell.

Keywords Langerhans cell histiocytosis $\cdot$ Major histocompatibility class II · HLA-DR · Immunophenotype

\section{Introduction}

Langerhans cell histocytosis (LCH) is usually a monoclonal proliferation of antigen presenting Langerhans cells (LCs) most commonly occurring in children in bone and soft tissue locations [1,2]. The disease can manifest as isolated bony or soft tissue lesions, multifocal, or multiorgan disease. The nuclei show the characteristic linear grooves, delicate chromatin, and abundant pale eosinophilic cytoplasm with admixed neutrophils, eosinophils, and multinucleated giant cells. In one series of LCH cases, the neoplastic cells of LCH were uniformly positive for $\mathrm{CD} 1 \mathrm{a}$ and $\mathrm{S} 100$ and frequently expressed CD68, with variable expression of CD45 [3]. Langerin (CD207), a component of Birbeck granules, is expressed in nearly all LCH cases [4-6]. Birbeck granules, which are characteristic of $\mathrm{LCH}$, are thought to be components of the endocytic recycling pathway [4-7]. BRAF V600E mutations, identical to those found in hairy cell leukemia, are present in $57 \%$ of LCH cases, raising the possibility of new therapeutic options for this disease $[8,9]$.

The antigen presenting major histocompatibility class II (MHCII) molecules are expressed on the surface of antigen presenting cells (APCs) of the immune system. In APCs, 
antigen loading of MHCII molecules occur in a specialized lysosomal compartment named MIIC, followed by transport of the MHCII-antigen complex to the cell surface for antigen presentation. LCH expresses molecules necessary for the antigen presenting function including the major histocompatibility class II molecules, such as HLA-DR, HLA-DP, and HLA-DQ [10-16]. However, the staining pattern in LCH has been minimally described with only one article reporting cytoplasmic staining for HLA-DR in six cases using confocal microscopy [17].

Because of our previous interest in abnormal patterns of MHCII expression in B cell malignancies, we determined to study the staining pattern of MHCII in normal dendritic and phagocytic cell populations as well as neoplastic LCH cells using the representative molecule HLA-DR, which is highly expressed in APCs. Herein, we describe a highly distinctive pattern of globular immunostaining for HLA-DR in the neoplastic cells of LCH. Our findings, if reproducible in other study sets, may have implications regarding stage of differentiation, pathogenesis, and possibly diagnosis of $\mathrm{LCH}$.

\section{Materials and methods}

Representative normal tissues known to contain Langerhans or histiocytic cell populations (bone marrow, liver, lung, lymph node, skin, spleen, thymus, and tonsil) were examined to provide a framework for interpreting the staining of $\mathrm{LCH}$ tumors. Formalin-fixed paraffin blocks of $44 \mathrm{LCH}$ samples were retrospectively procured from three study sites. Clinical history and diagnostic immunophenotype were obtained from the pathology reports (age, sex, location of biopsy, and IHC stain results from time of diagnosis) with Human Subjects Research approval at the University of Arizona.

Immunohistochemistry was performed on a Benchmark XT automated system [Ventana Medical Systems (VMS), Tucson, Arizona] with iView DAB detection. Monoclonal antibodies included anti-HLA-DR (Biogenex, 1:200) and anti-HLA-DR + DP + DQ (Abcam, 1:300). Anti-HLA-DR was performed on all samples while the anti-HLA-DR + DP + DQ assay was performed on four randomly selected samples for pattern verification. All cases were scored for intracellular location (cell surface, cytoplasmic granular, or cytoplasmic globular) and intensity (0 to $3+)$. Strong uniform intense staining was scored as a $3+$, moderate uniform staining as $2+$, and weak staining as $1+$, no staining as 0 . Cytoplasmic "globular" refers to one large globular stain accumulation adjacent to the nucleus, whereas cytoplasmic "granular" refers to multiple smaller, punctuate staining patterns throughout the cytoplasm. All normal tissues and $15 \mathrm{LCH}$ cases were scored by two pathologists (Redd and Rimsza) and the results compared.

\section{Results}

The pattern of staining varied between the cell populations in various tissues. HLA-DR cell surface expression was seen on all benign $B$ cell populations in all tissues, including germinal center B cells, mantle zone cells, interfollicular B cells, and marginal zone B cells in both lymph nodes and spleen. All interdigitating reticulum cells in the paracortical areas of lymph nodes were strongly positive in a cell surface pattern.
Fig. 1. HLA-DR staining in normal antigen presenting cells: a Low power view of lymph node shows staining of B cell nodules, very dark staining of scattered interdigitating dendritic cells in between the follicles, and negative staining of the $\mathrm{T}$ cells in the paracortex $(100 \times)$; b strong surface staining of mantle zone B cells $(400 \times)$; c strong surface staining of interdigitating cells (400×); $\mathbf{d}$ benign Langerhans cells in the skin $(400 \times)$ and inset (cropped and enlarged) showing granular cytoplasmic staining, but no large globules
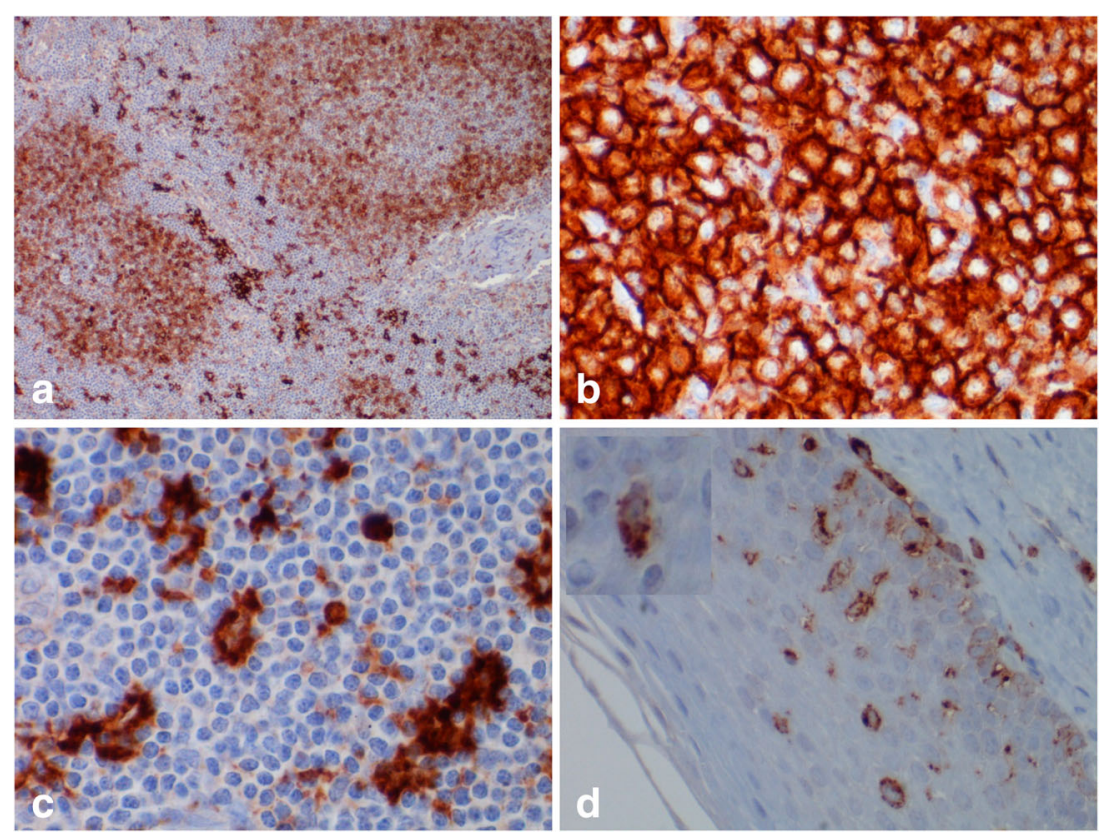
Table 1 Case series demographics, staining, and morphology

\begin{tabular}{|c|c|c|c|c|c|c|c|c|}
\hline Case & Location & Sex & $\begin{array}{l}\text { Age } \\
\text { (years) }\end{array}$ & $\begin{array}{l}\text { HLA-DR } \\
\text { pattern }\end{array}$ & Strength & Morphology & CD1a & S-100 \\
\hline 1 & Lymph node & $\mathrm{F}$ & 1 & CG, Glob & $3+$ & Classic & + & + \\
\hline 2 & Lymph node & M & 20 & CG, Glob & $3+$ & Sarcoma & NP & $\mathrm{NP}$ \\
\hline 3 & Bone (humerus) & M & 12 & CG, Glob & $3+$ & Classic & + & + \\
\hline 4 & Bone (rib) & M & 8 & CG, Glob & $3+$ & Classic & + & + \\
\hline 5 & Bone (skull) & $\mathrm{F}$ & 10 & CG, Glob & $3+$ & Classic & + & + \\
\hline 6 & Bone (skull) & M & 2 & CG, Glob & $3+$ & Classic & + & + \\
\hline 7 & Bone (skull) & $\mathrm{F}$ & $<1$ & CG, Glob & $3+$ & Classic & NP & NP \\
\hline 8 & Bone (vertebra) & M & 6 & CG, Glob & $3+$ & Classic & + & + \\
\hline 9 & Bone (skull) & $\mathrm{F}$ & 3 & CG, Glob & $3+$ & Classic & + & + \\
\hline 10 & Bone (unspecified) & M & 6 & CG, Glob & $1+$ & Classic & + & + \\
\hline 11 & Bone (skull) & M & 28 & CG, Glob & $3+$ & Classic & + & NP \\
\hline 12 & Bone (iliac) & $\mathrm{F}$ & 2 & CG, Glob & $3+$ & Classic & + & + \\
\hline 13 & Bone (skull) & M & 4 & CG, Glob & $3+$ & Classic & + & + \\
\hline 14 & Bone (ischial) & M & 4 & CG, Glob & $3+$ & Classic & + & + \\
\hline 15 & Bone (femur) & $\mathrm{F}$ & 9 & CG, Glob & $3+$ & Classic & + & + \\
\hline 16 & Bone (skull) & M & 40 & CG, Glob & $3+$ & Classic & + & + \\
\hline 17 & Bone (scapula) & M & 18 & CG, Glob & $3+$ & Classic & + & + \\
\hline 18 & Orbit & M & 7 & CG, Glob & $3+$ & Classic & + & + \\
\hline 19 & Orbit & $\mathrm{F}$ & 4 & CG, Glob & $3+$ & Classic & + & NP \\
\hline 20 & Orbit & M & 31 & CG, Glob & $3+$ & Sarcoma & NP & $\mathrm{NP}$ \\
\hline 21 & Orbit & $\mathrm{F}$ & 17 & CG, Glob & $3+$ & Classic & + & $\mathrm{NP}$ \\
\hline 22 & Lung & $\mathrm{F}$ & 39 & CG, Glob & $2+$ & Classic & + & + \\
\hline 23 & Lung & M & 18 & CG, Glob & $3+$ & Classic & + & + \\
\hline 24 & Lung & M & 40 & CG, Glob & $3+$ & Classic & + & + \\
\hline 25 & Lung & $\mathrm{F}$ & 8 & CG, Glob & $2+$ & Classic & + & + \\
\hline 26 & Lung & M & 39 & CG, Glob & $3+$ & Classic & + & + \\
\hline 27 & Lung & $\mathrm{F}$ & 42 & CG, Glob & $3+$ & Classic & + & + \\
\hline 28 & Lung & M & 54 & CG, Glob & $2+$ & Classic & + & + \\
\hline 29 & Lung & $\mathrm{F}$ & 41 & CG, Glob & $3+$ & Classic & + & + \\
\hline 30 & Lung & M & 51 & CG, Glob & $3+$ & Classic & + & + \\
\hline 31 & Lung & M & 62 & CG, Glob & $2+$ & Classic & + & + \\
\hline 32 & Skin & $\mathrm{F}$ & 54 & CG, Glob & $3+$ & Classic & + & - \\
\hline 33 & Skin & M & $<1$ & CG, Glob & $3+$ & Classic & + & $\mathrm{NP}$ \\
\hline 34 & Skin & M & 19 & CG, Glob & $2+$ & Classic & + & + \\
\hline 35 & Soft tissue, temple & $\mathrm{F}$ & 2 & CG, Glob & $3+$ & Classic & + & + \\
\hline 36 & Soft tissue, femur & M & 16 & CG, Glob & $3+$ & Classic & + & NP \\
\hline 37 & Soft tissue, femur & $\mathrm{F}$ & 7 & CG, Glob & $3+$ & Classic & + & + \\
\hline 38 & Mastoid contents & M & 3 & CG, Glob & $3+$ & Classic & + & + \\
\hline 39 & Liver & $\mathrm{F}$ & $<1$ & CG, Glob & $3+$ & Classic & + & + \\
\hline 40 & Spleen & M & 1 & CG, Glob & $3+$ & Classic & + & + \\
\hline 41 & Cervix & $\mathrm{F}$ & 2 & CG, Glob & $3+$ & Classic & + & + \\
\hline 42 & Sinus, frontal & M & 6 & CG, Glob & $3+$ & Classic & + & + \\
\hline 43 & Sinus, frontal & M & 6 & CG, Glob & $3+$ & Classic & $\mathrm{NP}$ & NP \\
\hline 44 & Diffuse, unspecified & M & $<1$ & CG, Glob & $3+$ & Classic & + & + \\
\hline
\end{tabular}

Case series demographics, staining patterns, and morphology for 44 cases of LCH. All 44 cases had an identical pattern of HLA-DR expression with the majority (43 cases) showing moderate to strong staining. Age, sex, location, $\mathrm{CD} 1 \mathrm{a}$, and $\mathrm{S} 100$ data were taken from original pathology reports

$C G$ cytoplasmic granular, Glob cytoplasmic globular, $N P$ not performed 
Plasma cells, wherever located, were negative. Surface staining was seen on a subset of T cells in various peripheral lymphoid tissues. Thymocytes were negative. Pulmonary macrophages and tingible body macrophages had a heterogeneous expression pattern, with a mixed surface and/or a granular cytoplasmic pattern of varying intensity. A granular cytoplasmic staining pattern alone (without surface expression) was seen in benign LCs in the skin and histiocytes in the spleen, lymph node, bone marrow, lung, liver, and thymus. None of the normal cell populations showed a globular perinuclear cytoplasmic pattern (see Fig. 1a-d). Concordance of staining results between the two pathologists for the normal tissues was $100 \%$.

The $44 \mathrm{LCH}$ patients' ages ranged from $<1$ to 62 years. Biopsy locations included skin, lung, soft tissue, orbit, spleen, lymph node, cervix, bone, and sinus. One sample reported systemic involvement. Sample details are listed in Table 1 and include IHC results from the diagnostic reports. Staining was scored by two pathologists with initial agreement in 14/15 cases ( $93 \%$ concordance). For the discordant case, discussion and resolution were performed at a multi-headed microscope and the consensus result used in further analysis. All $44 \mathrm{LCH}$ samples demonstrated cytoplasmic granular and globular staining pattern with no surface staining, which was a pattern not seen in any of the benign populations and therefore unique to the LCH cells. The majority of the LCH samples $(98 \%)$ demonstrated moderate to strong (2-3+) HLA-DR staining, with only one (decalcified) sample demonstrating weak staining (1+). In the majority of LCH cells, the MHCII globular aggregates were very large (see Fig. 2). This pattern was distinct from benign LC cells, which showed only a fine granular cytoplasmic staining pattern. We also applied an alternative monoclonal antibody recognizing a different, pan-MHCII, epitope on four samples, which showed the same cytoplasmic granular and globular staining pattern.

\section{Discussion}

This is the first report describing a distinct cytoplasmic granular and globular cytoplasmic staining pattern of MHCII in $\mathrm{LCH}$, using two different antibodies, a pattern not seen in representative normal tissues. Overall, there are very few studies describing MHCII expression in LCH since the initial reports in the 1980s. In those early papers, while MHCII was described as positive, the pattern of expression was not described. Similar to what we have reported, other authors, in 2001, also described cytoplasmic staining pattern for MHCII in six cases of LCH using confocal microscopy [17]. Thus, the current report is the largest case series and first using immunohistochemistry to describe this finding. The use of two different monoclonal antibodies, expected staining pattern in all types of benign APCs, phagocytic cells, and B cells, negativity in all non-hematopoietic cells, presence in samples from three different institutions with different fixation and processing methods, and corroborated by the reported confocal microscopy study, altogether support this as an authentic staining pattern.

Our observation of the absence of MHCII cell surface expression in LCH is consistent with previously published evidence of LCH cell immaturity $[17,18]$. Other studies have described high intracellular MHCII labeling in normal (benign) resident LCs with MHCII predominantly localized in the lysosome-like MIIC compartment, and in lower
Fig. 2. H\&E and HLA-DR staining in Langerhans cell histiocytosis: HLA-DR staining in Langerhans cell histiocytosis: a Hematoxylin and eosin stained section of LCH case \#1 (600×); b HLA-DR immunostain on case \#1 showing strong cytoplasmic globular staining (600×); c hematoxylin and eosin stained section of LCH case \#41; and d HLA-DR immunostain on case \#41 showing strong cytoplasmic globular staining $(600 \times)$
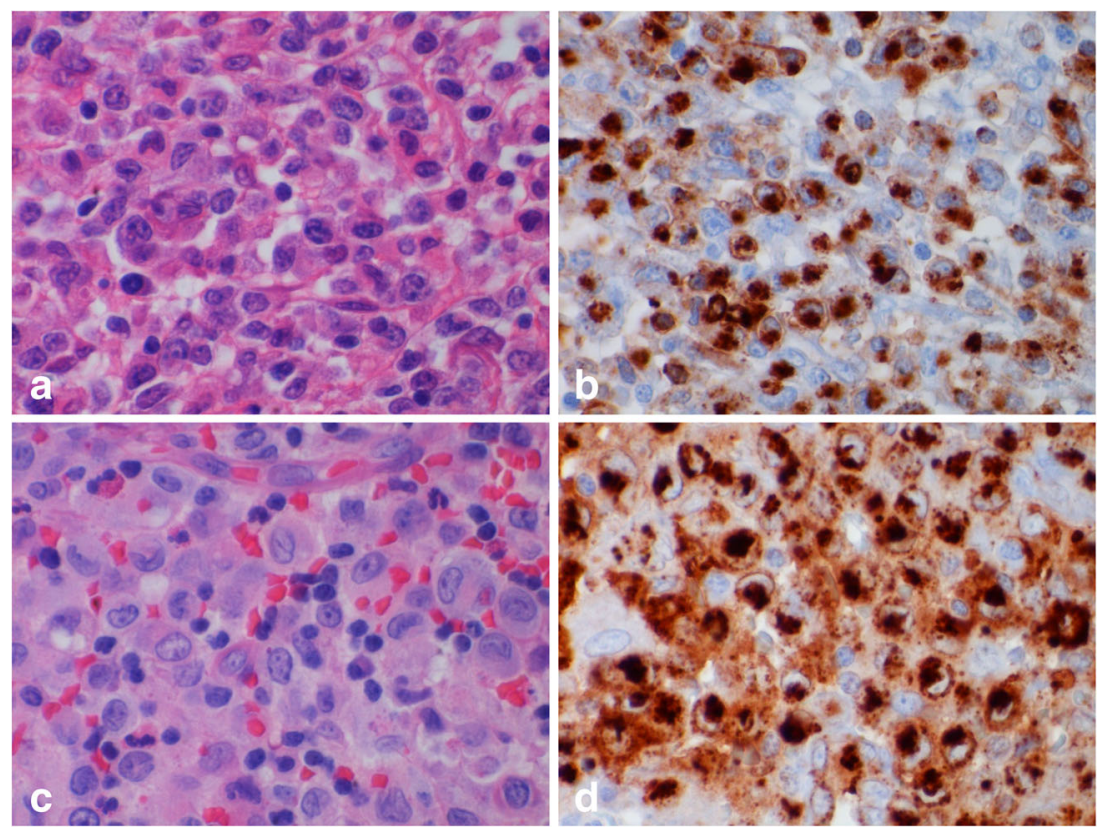
amounts on the membranes of the rough endoplasmic reticulum (RER). In contrast, it is reported that the cell surface, endosome-like structures, and the Birbeck granules are only slightly positive for MHCII in LCH $[19,20]$. Thus, the published literature does not support the presence of the accumulated MHCII protein within the Birbeck granules. The exact subcellular localization of the MHCII protein in LCH is unclear. Previously, using ultra-microscopy, Langfort et al. described a single case of pulmonary LCH with dilated RER in addition to Birbeck granules, however, there was no correlation with MHCII staining pattern [21]. Their report is in agreement with a single case in our own series that we were able to study by transmission electron microscopy that was suggestive of dilated RER (data not shown). A review of ultrastructural reports in the literature focuses on the description of the diagnostic Birbeck granules. The possibility that the MHCII is located in dilated RER in LCH requires further confirmation in a series of cases that have been of appropriately fixed and studied with electron or confocal microscopy.

Functionally, the antigen-presenting capacity of LCs is tightly controlled according to their maturation status [22]. The low surface expression of MHCII on resident epidermal LCs indicates they are not yet actively presenting antigen since inactive cells produce and hold MHCII intracellularly while awaiting antigen internalization, processing, and loading. Functionally, LCs residing in the skin are inefficient stimulators of $\mathrm{T}$ cell activation, whereas LCs which arrive in draining lymph nodes following skin sensitization are highly immunogenic with a significant increase in MHCII surface expression, consistent with phenotypic maturation and the acquisition of antigen-presenting function [23-25]. In immature cells, MHCII accumulates in lysosome-like organelles colocalizing with resident lysosomal proteins such as HLADM and hydrolytic enzymes [26, 27] and corresponds to the granular staining that we observed throughout the cytoplasm in the benign LC cells. Upon environmental signal induced maturation, MHCII-antigen complexes move via peripheral non-lysosomal compartments to the cell surface, which correlates to the pattern we observed in mature APCs in lymph nodes and on B cells [28]. It is therefore possible to conclude that the cytoplasmic staining pattern in $\mathrm{LCH}$ reflects the immature/antigen-inexperienced status of the malignant cell in $\mathrm{LCH}$.

On a practical level, while a characteristic immunophenotype is known for LCH including expression of CD1a and S100, not all cases express both antigens. In addition, CD207 (Langerin) is not universally used in diagnostic pathology laboratories. Furthermore, we performed a limited investigation on some histologic mimics including one dermatopathic lymphadenopathy, two cases of Rosai-Dorfman disease, one follicular dendritic cell tumor, one interdigitating dendritic cell tumor, and two histiocytic sarcomas, which did not show the cytoplasmic globular staining pattern observed in LCH (data not shown). As such, the distinct HLA-DR staining pattern in LCH may also be a useful tool for diagnosis; however more extensive studies are necessary. In summary, MHCII, with its highly distinctive staining pattern, could possibly, with additional verification in other patient cohorts, be useful as an additional marker for identifying neoplastic Langerhans cells, contribute observational data relevant to the stage of differentiation of the LCH cells, and add to our understanding of the biology of this rare disease.

\section{Compliance with ethical standards}

Conflict of interest The authors declare that they have no conflict of interest.

Funding The study was funded by Hope Foundation (Monika Schmelz).

\section{References}

1. Jaffe ES (2001) WHO classification of tumours, pathology and genetics, tumours of haematopoietic and lymphoid tissues. IARC Press, Lyon

2. Willman CL, Busque L, Griffith BB, Favara BE, McClain KL, Duncan MH, et al. (1994) Langerhans'-cell histiocytosis (histiocytosis X)-a clonal proliferative disease. N Engl J Med 331(3):154-160

3. Pileri SA, Grogan TM, Harris NL, Banks P, Campo E, Chan JK, et al. (2002) Tumours of histiocytes and accessory dendritic cells: an immunohistochemical approach to classification from the International Lymphoma Study Group based on 61 cases. Histopathology 41(1):1-29

4. Chikwava K, Jaffe R (2004) Langerin (CD207) staining in normal pediatric tissues, reactive lymph nodes, and childhood histiocytic disorders. Pediatr Dev Pathol 7(6):607-614

5. Mc DR, Ziylan U, Spehner D, Bausinger H, Lipsker D, Mommaas M, et al. (2002) Birbeck granules are subdomains of endosomal recycling compartment in human epidermal Langerhans cells, which form where Langerin accumulates. Mol Biol Cell 13(1): 317-335

6. Sholl LM, Hornick JL, Pinkus JL, Pinkus GS, Padera RF (2007) Immunohistochemical analysis of langerin in langerhans cell histiocytosis and pulmonary inflammatory and infectious diseases. Am J Surg Pathol 31(6):947-952

7. Thepaut M, Valladeau J, Nurisso A, Kahn R, Arnou B, Vives C, et al. (2009) Structural studies of langerin and birbeck granule: a macromolecular organization model. Biochemistry 48(12):2684-2698

8. Badalian-Very G, Vergilio JA, Degar BA, MacConaill LE, Brandner B, Calicchio ML, et al. (2010) Recurrent BRAF mutations in Langerhans cell histiocytosis. Blood 116(11):1919-1923

9. Haroche J, Cohen-Aubart F, Emile JF, Arnaud L, Maksud P, Charlotte F, et al. (2013) Dramatic efficacy of vemurafenib in both multisystemic and refractory erdheim-Chester disease and Langerhans cell histiocytosis harboring the BRAF V600E mutation. Blood 121(9): 1495-1500

10. Bos JD, Sillevis Smitt JH, Krieg SR, Bakker PM, Vos GD, van Zaane DJ (1984) Acute disseminated histiocytosis-X: in situ immunophenotyping with monoclonal antibodies. J Cutan Pathol 11(1):59-64 
11. Flint A, Lloyd RV, Colby TV, Wilson BW (1986) Pulmonary histiocytosis X. immunoperoxidase staining for HLA-DR antigen and S100 protein. Arch Pathol Lab Med 110(10):930-933

12. McMillan EM, Humphrey GB, Stoneking L, Strauss LC, Civin CI, Abo T, et al. (1986 Mar) Analysis of histiocytosis X infiltrates with monoclonal antibodies directed against cells of histiocytic, lymphoid, and myeloid lineage. Clin Immunol Immunopathol 38(3): 295-301

13. Ornvold K, Ralfkiaer E, Carstensen H (1990) Immunohistochemical study of the abnormal cells in Langerhans cell histiocytosis (histiocytosis x). Virchows Arch A Pathol Anat Histopathol 416(5): 403-410

14. Ralfkiaer E, Stein H, Ralfkiaer N, Hou-Jensen K, Mason DY (1985) Normal and neoplastic Langerhans cells: phenotypic comparison with other types of macrophages. Adv Exp Med Biol 186: 1009-1015

15. Slater DN, Rooney N, Harrington C, Tucker WF, Beck S, Walker AE, et al. (1984) Generalized histiocytosis X in the elderly: a light and electron microscope and monoclonal antibody study. Histopathology 8(6):927-936

16. dV v, Besselaar-Dingjan G, MR D, CJ M (1983) Analysis of largecell lymphomas using monoclonal and heterologous antibodies. $\mathrm{J}$ Clin Pathol 36(1):44-50

17. Geissmann F, Lepelletier Y, Fraitag S, Valladeau J, Bodemer C, Debre M, et al. (2001) Differentiation of Langerhans cells in Langerhans cell histiocytosis. Blood 97(5):1241-1248

18. Egeler RM, van Halteren AG, Hogendoorn PC, Laman JD, Leenen PJ (2010) Langerhans cell histiocytosis: fascinating dynamics of the dendritic cell-macrophage lineage. Immunol Rev 234(1):213-232

19. Arkema JM, Schadee-Eestermans IL, Broekhuis-Fluitsma DM, Hoefsmit EC (1991) Localization of class II molecules in storage vesicles, endosomes and lysosomes in human dendritic cells. Immunobiology 183(5):396-407
20. Kleijmeer MJ, Oorschot VM, Geuze HJ (1994) Human resident langerhans cells display a lysosomal compartment enriched in MHC class II. J Investig Dermatol 103(4):516-523

21. Langfort R, Radzikowska E, Czarnowska E, Wiatr E, Grajkowska W, Blasinska-Przerwa K, et al. (2009) Langerhans cell sarcoma with pulmonary manifestation, mediastinum involvement and bronchoesophageal fistula. A rare location and difficulties in histopathological diagnosis. Pneumonol Alergol Pol 77(3):327-334

22. Mellman I, Steinman RM (2001) Dendritic cells: specialized and regulated antigen processing machines. Cell 106(3):255-258

23. Cumberbatch M, Gould SJ, Peters SW, Kimber I (1991) MHC class II expression by Langerhans' cells and lymph node dendritic cells: possible evidence for maturation of Langerhans' cells following contact sensitization. Immunology 74(3):414-419

24. Schuler G, Steinman RM (1985) Murine epidermal Langerhans cells mature into potent immunostimulatory dendritic cells in vitro. J Exp Med 161(3):526-546

25. Shimada S, Caughman SW, Sharrow SO, Stephany D, Katz SI (1987) Enhanced antigen-presenting capacity of cultured Langerhans' cells is associated with markedly increased expression of Ia antigen. J Immunol 139(8):2551-2555

26. Cella M, Engering A, Pinet V, Pieters J, Lanzavecchia A (1997) Inflammatory stimuli induce accumulation of MHC class II complexes on dendritic cells. Nature 388(6644):782-787

27. Pierre P, Turley SJ, Gatti E, Hull M, Meltzer J, Mirza A, et al. (1997) Developmental regulation of MHC class II transport in mouse dendritic cells. Nature 388(6644):787-792

28. Turley SJ, Inaba K, Garrett WS, Ebersold M, Unternaehrer J, Steinman RM, et al. (2000) Transport of peptide-MHC class II complexes in developing dendritic cells. Science 288(5465): $522-527$ 